\title{
Crystal growth in floating zone with phase change and thermo-solutal convections
}

\author{
B. Xiong and W.R. Hu \\ Institute of Mechanics, Chinese Academy of Sciences, 15 Zhong Guan Cun Road, Beijing 100080, People's Republic of China
}

Received 28 April 1992; manuscript received in final form 16 June 1992

Floating zone crystal growth in microgravity environment is investigated numerically by a finite element method for semiconductor growth processing, which involves thermocapillary convection, phase change convection, thermal diffusion and solutal diffusion. The configurations of phase change interfaces and distributions of velocity, temperature and concentration fields are analyzed for typical conditions of pulling rates and segregation coefficients. The influence of phase change convection on the distribution of concentration is studied in detail. The results show that the thermocapillary convection plays an important role in mixing up the melt with dopant. The deformations of phase change interfaces by thermal convection-diffusion and pulling rods make larger variation of concentration field in comparison with the case of plane interfaces.

\section{Introduction}

The floating zone crystal growth technique, invented by Pfann [1], has been widely used to purify high quality crystals. Like other techniques of crystal growth, there are convections induced by various mechanisms in the medium of fluid, and the quality of the crystal, such as the purification, homogeneity and segregation, will be influenced [1-4]. Convections in melting zone may be induced, under no matter what gravity condition, by buoyancy on the ground of gravity environment or thermocapillary and/or solutocapillary effect in low- or microgravity environment $[2,18]$. In addition, the phase change convection exists in real processing of crystal growth [15,21]. Therefore, the coupling of phase change near solidification and melting interfaces, together with convections and diffusions in melting zone $[3,17,18,22]$, is significantly influenced and should be simulated by theoretical models.

Striations in the crystals grown have been found in multifarious techniques and have been interpreted by both natural convection and thermocapillary convection $[2,18]$. The influence of convection on concentration distribution was pointed out since the invention of the floating zone technique [1,5]. The influence of convection on longitudinal segregation, which is parallel to the growing direction, was studied by Burton, Prim and Slichter [5], and then, the so-called BPS model was developed extensively [6-10], particularly for a semi-quantitative description of one-dimensional concentration distribution. On the other hand, the segregation in the lateral direction cannot be treated by a one-dimensional model involving the influence of convection [2].

Many papers have been published to consider the lateral segregation in the model without phase change [4,7-9], and the melting/solidification interfaces have been assumed to be planar solid boundaries without mass transfer. A model involving melting and solidification interfaces was calculated numerically for the crystal growth in a vertical Bridgman system, in consideration of the influence of natural convection and the segregation in two directions [3]. The results show that the usual approximation of diffusion layer for the dopant distribution adjacent to the crystal needs to be improved [3].

Recently, crystal growth in the floating zone has attracted more attention from both experimental and theoretical sides due to the development of microgravity science [11-17]. Natural convection and 
Marangoni convection have been investigated extensively [11-18]. The results have shown that Marangoni convection driven by the gradient of surface tension in the free surface of the liquid bridge is most important in the case of low- or microgravity [11,15]. Nevertheless, convection which induces lateral segregation always exists. The thermal and solutal capillary convections and double diffusions in the model of half floating zone without phase change have been studied $[4,19,23]$. Two sorts of concentration boundary conditions were discussed in ref. [4]: one was the usual BPS condition and the other was the concentration conservation condition. Since the curvature of the interfaces will also induce lateral segregation, the assumption on planar interface should be considered in concentration distribution in a complete model. Interaction between thermocapillary convection and melting/solidification phase change convection has been discussed $[17,18]$ and the concentration conservation model with phase change was analyzed elsewhere [20].

The main purpose of the present paper is to study the concentration field and the segregation in consideration of the influence of thermocapillary convection together with melting/solidification phase change convection in the floating zone under microgravity condition. A FEM method is used to simulate phase change interfaces, fluid flow isotherms and concentration fields in the melting zone, and isotherms in the solid zones for different pulling rates and for different segregation coefficients under microgravity condition. The results show that the phase change interfaces obviously influence the concentration distribution in the liquid bridge. A comparison is made of the results of previous work $[4,11,20]$ and those of the present paper. The physical model and mathematical expressions are given in section 2 . The numerical methods are presented in section 3. Section 4 gives the numerical results of flow patterns and concentration distributions of the convection. The last section gives the conclusions.

\section{Description of the model}

In the floating zone technique of crystal growth, a feed rod is pulled toward the high temperature zone of the crystal melt heated by a heater of radiation ring. The resolidified crystal is obtained when it is pulled out of the melting zone, as shown in fig. 1. The dopant impurity in the feed and melt zone is assumed to be low for typical semiconductor processing. The liquid bridge of the melt zone is held by surface tension and locates between the upper rod of the single crystal and the lower rod of the multi-crystal.

To construct a complete model of the full floating zone, we will consider at least three regions, i.e., the region of the liquid bridge, the solid region of the feed rod and the solid region of the single-crystal rod. The solutions in each region are coupled with those in the other regions. However, the solutions of the full floating zone problem may be obtained by matching the solutions in the three regions with the boundary conditions which can be satisfied by an iterative procedure. For the sake of simplicity, we assume that the problem is axisymmetric, laminar and steady. The cylindrical coordinate system is used and the plane $z=0$ is fixed in the heater plane, which is considered to be a thermal radiation ring. The thermophysical parameters of the melt and the solid are nearly the same as given in refs. [17,20]. The single crystal and the feed rods have the same diameter $r_{0}$ and move with the same rate $v_{\mathrm{p}}$ in the $z$-direction.

Free surface deformation is ignored at the first step, and $r_{0}$ is the radius of the liquid bridge. The governing equations are mass conservation, momentum conservation, energy conservation and diffusion equation under suitable boundary conditions, the same as those in ref. [20] for a steady process, except for the concentration boundary condition. To make the results more general, dimensionless parameters are introduced as follows:

$$
\begin{aligned}
& \zeta=\frac{z}{r_{0}}, \quad \xi=\frac{r}{r_{0}}, \quad U=\frac{u}{u_{0}}, \quad W=\frac{w}{u_{0}}, \quad P=\frac{p}{\rho u_{0}^{2}}, \\
& \Theta_{i}=\frac{T_{i}-T_{\mathrm{m}}}{T_{0}-T_{\mathrm{m}}} \quad(i=1,2), \quad \Theta=\frac{T-T_{\underline{\mathrm{m}}}}{T_{0}-T_{\mathrm{m}}}, \quad C=\frac{c}{c_{\mathrm{s}}},
\end{aligned}
$$




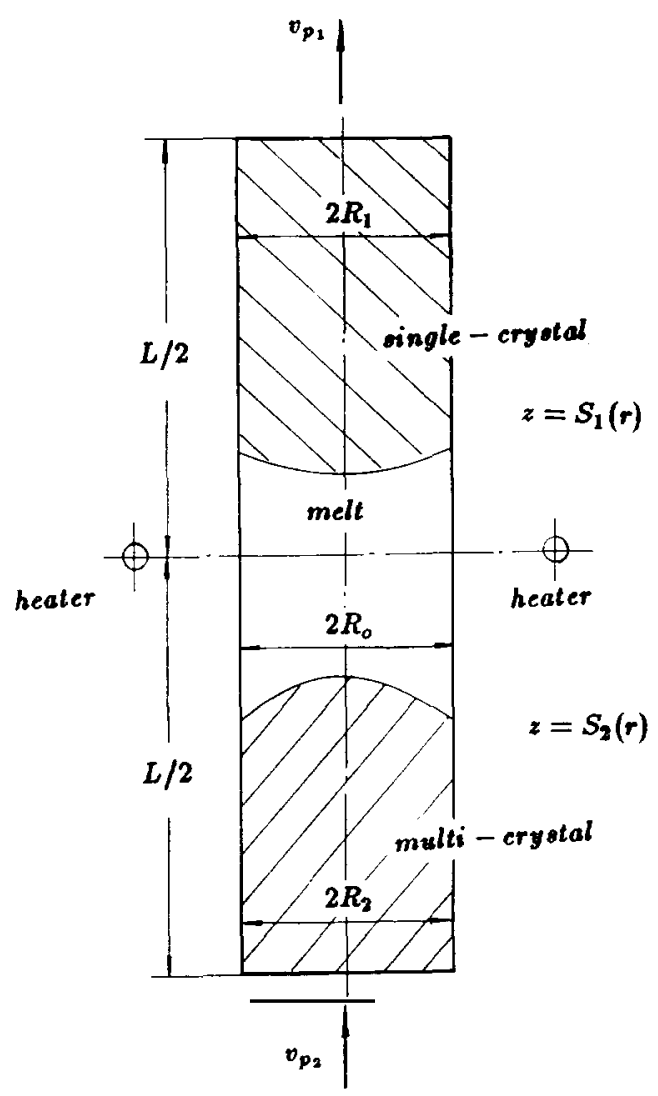

Fig. 1. Schematic diagram of floating zone system model.

where the subscript zero denotes the typical value; $T_{0}$ and $T_{\mathrm{m}}$ are the heating temperature and melting point, respectively, $(u, w)$ is the flow velocity in $(r, z)$ direction, $p, T$ and $c$ are the pressure, temperature and concentration in the liquid bridge, respectively, $T_{1}$ and $T_{2}$ denote the temperatures in the single crystal region and feed region, respectively, and the constant $c_{\mathrm{s}}$ is the concentration of the feed rod. The characteristic velocity $u_{0}$ remains to be determined. The appropriate characteristic velocity in the thermocapillary problem may be the characteristic velocity

$$
u_{0}=\left|\sigma_{\tau}\right|\left(T_{0}-T_{\mathrm{m}}\right) / \mu,
$$

driven by the gradient of surface tension, where $\sigma_{\tau}$ and $\mu$ are the thermal coefficient of surface tension and the melt viscosity respectively. According to these relationships, the dimensionless equations may be written as follows [20]:

$$
\operatorname{Pe}_{i} \frac{\partial \Theta_{i}}{\partial \zeta}=\frac{\partial^{2} \Theta_{i}}{\partial \zeta^{2}}+\frac{\partial^{2} \Theta_{i}}{\partial \xi^{2}}+\frac{1}{\xi} \frac{\partial \Theta_{i}}{\partial \xi} \quad(i=1,2)
$$

for the regions of solid rods, and

$$
\frac{\partial U}{\partial \xi}+\frac{U}{\xi}+\frac{\partial W}{\partial \zeta}=0,
$$




$$
\begin{aligned}
& \operatorname{Re}\left(U \frac{\partial U}{\partial \xi}+W \frac{\partial U}{\partial \zeta}\right)=-\operatorname{Re} \frac{\partial P}{\partial \xi}+\frac{\partial^{2} U}{\partial \zeta^{2}}+\frac{\partial^{2} U}{\partial \xi^{2}}+\frac{1}{\xi} \frac{\partial U}{\partial \xi}-\frac{U}{\xi^{2}}, \\
& \operatorname{Re}\left(U \frac{\partial W}{\partial \xi}+W \frac{\partial W}{\partial \zeta}\right)=\operatorname{Bo} \Theta-\operatorname{Re} \frac{\partial P}{\partial \zeta}+\frac{\partial^{2} W}{\partial \zeta^{2}}+\frac{\partial^{2} W}{\partial \xi^{2}}+\frac{1}{\xi} \frac{\partial W}{\partial \xi}, \\
& \operatorname{Ma}\left(U \frac{\partial \Theta}{\partial \xi}+W \frac{\partial \Theta}{\partial \zeta}\right)=\frac{\partial^{2} \Theta}{\partial \zeta^{2}}+\frac{\partial^{2} \Theta}{\partial \xi^{2}}+\frac{1}{\xi} \frac{\partial \Theta}{\partial \xi}, \\
& \operatorname{Pe}_{\mathrm{s}}\left(U \frac{\partial C}{\partial \xi}+W \frac{\partial C}{\partial \zeta}\right)=\frac{\partial^{2} C}{\partial \zeta^{2}}+\frac{\partial^{2} C}{\partial \xi^{2}}+\frac{1}{\xi} \frac{\partial C}{\partial \xi},
\end{aligned}
$$

for the region of liquid bridge. The dimensionless boundary conditions are

$$
\begin{aligned}
& \xi=0, \quad \zeta \notin\left(S_{2}(0), S_{1}(0)\right): \quad \frac{\partial \Theta_{1}}{\partial \xi}=0, \quad \frac{\partial \Theta_{2}}{\partial \xi}=0, \\
& \left.\xi=1, \quad \zeta \notin\left(S_{2}(1), S_{1}(1)\right): \quad-\frac{\partial \Theta_{i}}{\partial \xi}=\mathrm{Bi}_{i}\left[\left(\Theta_{i}+T_{\mathrm{m}}^{*}\right)^{4}-T_{0}^{* 4}\right)\right] f(\zeta) \quad(i=1,2), \\
& \zeta=S_{i}(\xi), \quad \xi \in(0,1): \quad \Theta_{i}=0 \quad(i=1,2), \\
& \zeta= \pm L_{i}, \quad \xi \in(0,1): \quad \Theta_{i}=\Theta_{\infty i} \quad(i=1,2),
\end{aligned}
$$

for the regions of solid rods, and

$$
\begin{aligned}
\xi=0, \quad \zeta \in\left(S_{2}(0), S_{1}(0)\right): & \frac{\partial U}{\partial \xi}=0, \quad \frac{\partial W}{\partial \xi}=0, \quad \frac{\partial \Theta}{\partial \xi}=0, \quad \frac{\partial C}{\partial \xi}=0, \\
\xi=1 \quad \zeta \in\left(S_{2}(1), S_{1}(1)\right): \quad & U=0, \quad \frac{\partial W}{\partial \xi}=-\frac{\partial \Theta}{\partial \zeta}-\delta_{\mathrm{s}} \frac{\partial C}{\partial \zeta}, \\
& \partial C / \partial \xi=0, \\
& \left.-\partial \Theta / \partial \xi=\operatorname{Bi}\left[\left(\Theta+T_{\mathrm{m}}^{*}\right)^{4}-T_{0}^{* 4}\right)\right] f(\zeta),
\end{aligned}
$$

$\zeta=S_{1}(\xi), \quad \xi \in(0,1)$

$$
\begin{aligned}
& U=\frac{\epsilon_{1} V_{\mathrm{p}_{1}} \mathrm{~d} S_{1} / \mathrm{d} \xi}{1+\left(\mathrm{d} S_{1} / \mathrm{d} \xi\right)^{2}}, \\
& W=\frac{\epsilon_{1} V_{\mathrm{p}_{1}}}{1+\left(\mathrm{d} S_{1} / \mathrm{d} \xi\right)^{2}}-V_{\mathrm{p}_{1}}, \\
& \Theta=0 . \\
& \partial C / \partial n=\operatorname{Pe}_{\mathrm{s}} V_{\mathrm{p}_{n}}\left(1-k_{0}\right) C,
\end{aligned}
$$

$\zeta=S_{2}(\xi), \quad \xi \in(0,1)$

$$
\begin{aligned}
& U=\frac{\epsilon_{2} V_{\mathrm{p}_{2}} \mathrm{~d} S_{2} / \mathrm{d} \xi}{1+\left(\mathrm{d} S_{2} / \mathrm{d} \xi\right)^{2}}, \\
& W=\frac{\epsilon_{2} V_{\mathrm{p}_{2}}}{1+\left(\mathrm{d} S_{2} / \mathrm{d} \xi\right)^{2}}-V_{\mathrm{p}_{2}}, \\
& \Theta=0, \\
& \partial C / \partial n=-\mathrm{Pe}_{\mathrm{s}} V_{\mathrm{p}_{n}}\left(C-C_{\mathrm{s}}\right),
\end{aligned}
$$


for the region of liquid bridge, where $\zeta=S_{1}(\xi)$ and $\zeta=S_{2}(\xi)$ are the melting boundary and the solidification boundary of phase change interfaces respectively. The angular coefficient of thermal radiation, $f(\zeta)$, is assumed to be a Gaussian distribution, such as $f(\zeta)=f_{0} \mathrm{e}^{-\lambda \zeta^{2}}$. In order to obtain an appropriate size of the melting zone, $\lambda=2$ is adopted and $f_{0}$ is combined into the Biot number Bi. The dimensionless Stefan conditions become

$$
\frac{\partial \Theta_{i}}{\partial n}-\delta_{i} \frac{\partial \Theta}{\partial n}= \pm \frac{\mathrm{St}_{i}\left|V_{\mathrm{p}_{i}}\right|}{\sqrt{1+\left(\mathrm{d} S_{i}^{-} / \mathrm{d} \xi\right)^{2}}} \quad(i=1,2)
$$

The following non-dimensional parameters have been introduced

$$
\begin{aligned}
& \mathrm{Re}=\frac{r_{0} u_{0}}{\nu}, \quad \mathrm{Ma}=\frac{r_{0} u_{0}}{\alpha}, \quad \mathrm{Bo}=\frac{\beta g r_{0}^{2}\left(T_{0}-T_{\mathrm{m}}\right)}{\nu u_{0}}, \quad \mathrm{Pe}_{i}=\frac{v_{\mathrm{p}_{i}} r_{0}}{\alpha_{i}}, \\
& \delta_{i}=\frac{K}{K_{i}}, \quad \mathrm{St}_{i}=\frac{\Delta H_{i} \rho r_{0} u_{0}}{\overline{K_{i}\left(T_{0}-T_{\mathrm{m}}\right)}}, \quad T_{\mathrm{m}}^{*}=\frac{T_{\mathrm{m}}}{T_{0}-T_{\mathrm{m}}}, \\
& T_{0}^{*}=\frac{T_{0}}{T_{0}-T_{\mathrm{m}}}, \quad V_{\mathrm{p}_{i}}=\frac{v_{\mathrm{p}_{i}}}{u_{0}}, \quad \mathrm{Bi}_{i}=\frac{f_{0} r_{0} \epsilon_{i}^{*} \sigma^{*}\left(T_{0}-T_{\mathrm{m}}\right)^{3}}{K_{i}}, \\
& \mathrm{Bi}=\frac{f_{0} r_{0} \epsilon^{*} \sigma^{*}\left(T_{0}-T_{\mathrm{m}}\right)^{3}}{-}, \quad \mathrm{Pe}_{\mathrm{s}}=\frac{r_{0} u_{0}}{D}, \quad \delta_{\mathrm{s}}=\frac{\sigma_{\mathrm{s}} c_{\mathrm{s}}}{\sigma_{\tau} \Delta T} \quad(i=1,2),
\end{aligned}
$$

where $\alpha, \alpha_{1}$ and $\alpha_{2}$ are the thermal diffusivities and $K, K_{1}$ and $K_{2}$ are the thermal conductivity of the melt, single crystal and feed materials, respectively; $\Delta H$ is the heat of fusion; $\epsilon^{*}, \epsilon_{1}^{*}$ and $\epsilon_{2}^{*}$ are the emissivities of the melt, crystal and feed, respectively; $\sigma^{*}$ is the Stefan-Boltzmann constant; $D$ is the dopant diffusivity in the melt; $k_{0}$ is the dopant segregation coefficient;

$$
V_{\mathrm{p}_{n}}=V_{\mathrm{p}} /\left[1+(\mathrm{d} S / \mathrm{d} \xi)^{2}\right]^{0.5}
$$

$\boldsymbol{n}$ is the normal unit vector to interfaces which directs to melt region. The solution in the three regions will satisfy the matching conditions at the phase change interfaces $\zeta=S_{1}(\xi)$ and $\zeta=S_{2}(\xi)$, i.e. conditions (2.10) and (2.16)-(2.24). The so-called Stefan condition, derived from the energy conservation across the interfaces, is used together with other boundary conditions to determined the interface shapes.

It is difficult to grip the essential of the problems since there are so many controlling parameters. But our object is to understand the influence of convection and of phase change interfaces on the segregation. Therefore, the pulling rate $V_{\mathrm{p}_{i}}$ and segregation coefficient $K_{0}$ will be analyzed especially in the present paper.

\section{Numerical simulation}

To solve the problem in fluid region, we introduced as usual the stream function $\psi$ defined as follows

$$
W=\frac{1}{\xi} \frac{\partial(\xi \psi)}{\partial \xi}, \quad U=-\frac{\partial \psi}{\partial \zeta},
$$


and the vorticity

$$
\Omega=\frac{\partial U}{\partial \zeta}-\frac{\partial W}{\partial \xi} .
$$

The equations in the liquid bridge may then be expressed in relations of stream function-vorticity instead of velocity components $U, W$ and $P[17,20]$. An "up-wind" finite element scheme improved from the Galerkin finite element method is applied to overcome the difficulties caused by the convection terms in some cases with relative larger value of parameter [24]. In an element, the variables are the linear combinations of node values, expressed as the follows:

$$
\begin{aligned}
& \psi^{(e)}=N_{i}^{(e)} \psi_{i}^{(e)}, \quad \Omega^{(e)}=N_{i}^{(e)} \Omega_{i}^{(e)}, \quad C^{(e)}=N_{i}^{\left(e^{e}\right)} C_{i}^{\left(e^{e}\right)}, \\
& \Theta^{(e)}=N_{i}^{(e)} \Theta_{i}^{(e)}, \quad \Theta_{1}^{(e)}=N_{i}^{(e)} \Theta_{1_{i}^{(e)}}, \quad \Theta_{2}^{(e)}=N_{i}^{(e)} \Theta_{2_{i}^{(e)}}^{(i=1,2,3,4),}
\end{aligned}
$$

where the superscript $(e)$ denotes the element $(e) ; N_{i}^{(e)}$ are the shape functions. With local $x, y$ coordinates normalizing a variation from -1 to +1 , we can write

$$
N_{i}^{(e)}=N_{i}^{*}(x) N_{i}^{*}(y)=\frac{1}{2}\left(1+s_{i}^{x} x\right) \times \frac{1}{2}\left(1+s_{i}^{y} y\right) \quad(i=1,2,3,4),
$$

where

$$
s_{i}^{x}=\left\{\begin{array}{ll}
+1, & i=2,3, \\
-1, & i=1,4,
\end{array} \quad s_{i}^{y}= \begin{cases}+1, & i=3,4 \\
-1, & i=1,2 .\end{cases}\right.
$$

Similarly, the weighting function can be written as

$$
\begin{aligned}
G_{i}^{(e)} & =\left[N_{i}^{*}(x)+\frac{3}{4} \alpha_{i j}(1-x)(1+x)\right]\left[N_{i}^{*}(y)+\frac{3}{4} \beta_{i j}(1-y)(1+y)\right] \\
& =N_{i}^{*}(x) N_{i}^{*}(y)+F_{i}^{(e)}=N_{i}^{(e)}+F_{i}^{(e)} \quad(i=1,2,3,4) .
\end{aligned}
$$

$F_{i}^{(e)}$ limits to zero for the Galerkin method. Substituting (3.3) and (3.6) into the weighting residual equations of the governing equations, we can obtain the discretization equations. It is convenient to consider the iteration procedure as a "pseudo-growth process" by the balance of latent heat with the heat fluxes across the interfaces. The usual unsteady Stefan conditions about the phase change interfaces in floating zone crystal growth are:

$$
\pm K_{i} \frac{\partial T_{i}}{\partial n} \mp K \frac{\partial T}{\partial n}=\Delta H_{i} \rho\left(v_{\mathrm{p}_{n}}-\frac{\mathrm{d} s_{i}}{\mathrm{~d} t} \cos \alpha\right) \quad(i=1,2)
$$

in the dimensional form, or

$$
\lambda_{i}=\frac{\mathrm{d} S_{i}}{\mathrm{~d} t}=\left(\frac{\partial \Theta_{i}}{\partial \zeta}-\delta_{i} \frac{\partial \Theta}{\partial \zeta}\right)\left[1+\left(\frac{\mathrm{d} S_{i}}{\mathrm{~d} \xi}\right)^{2}\right] \mathrm{St}_{i}^{-1}+\left|V_{\mathrm{p}_{i}}\right| \quad(i=1,2)
$$

in the dimensionless form. So we can obtain $S_{i}^{n+1}=S_{i}^{n}+\lambda_{i} \Delta t$. The pseudo-time interval $\Delta t$ is selected to satisfy the convergence condition of the problem. A proper solution is obtained when $\mathrm{d} S_{i} / \mathrm{d} t$ tends to zero while the governing equations are satisfied in their respective fields. In a certain parameter range, the solution could be obtained, which could give the convergent interface positions.

The convergent solution could be obtained by iterative method due to adjustment of the solidification boundary. First, we assume two interfaces of phase change and solve the governing equations under the fixed boundary of given interface. The surface solutal capillary effect is ignored here due to its very small effect [4]. So the concentration distribution is uncoupled with flow, temperature and interface positions. 
Table 1

Values of the thermophysical properties of silicon and operating

\begin{tabular}{ll}
\hline Property & Value \\
\hline Density $\rho$ & $2.44 \times 10^{3} \mathrm{~kg} / \mathrm{m}^{3}$ \\
Heat capacity $C_{p}$ & $1.049 \times 10^{3} \mathrm{~J} / \mathrm{kg} \cdot \mathrm{K}$ \\
Thermal conductivity $K$ & $4.30 \times 10^{1} \mathrm{~W} / \mathrm{m} \cdot \mathrm{K}$ \\
Dopant diffusivity $D$ & $2.56 \times 10^{-7} \mathrm{~m}^{2} / \mathrm{s}$ \\
Melting point $T_{\mathrm{m}}$ & $1688 \mathrm{~K}$ \\
Heating temperature $T_{0}$ & $1668+844 \mathrm{~K}$ \\
Heat of fusion $\Delta H$ & $1.803 \times 10^{6} \mathrm{~J} / \mathrm{kg}$ \\
Heat coefficient of surface tension $\sigma_{\tau}$ & $-1.0 \times 10^{-4} \mathrm{~kg} / \mathrm{s}^{2} \cdot \mathrm{K}$ \\
Melt viscosity $\mu$ & $8.25 \times 10^{-4} \mathrm{~kg} / \mathrm{m} \cdot \mathrm{s}$ \\
Emissivity $\epsilon^{*}$ & 0.5 \\
Rod radius $r_{0}$ & $5 \times 10^{-3} \mathrm{~m}$ \\
Ampoule length $L \times 2$ & $8.0 \times 10^{-2} \mathrm{~m}$ \\
Rod temperature at the end $T_{\infty}$ & $1304 \mathrm{~K}$ \\
Pulling rate $\iota_{\mathrm{p}}$ & $0-10^{-5} \mathrm{~m} / \mathrm{s}$ \\
\hline
\end{tabular}

Then, we adjust the interface positions by eq. (3.8), and repeat the iterative process until the convergence condition, $\mathrm{d} S_{i} / \mathrm{d} t \rightarrow 0$, is satisfied. The iterative procedure is successful for the problem.

The numerical simulation is applied especially for the silicon crystal growth. The thermophysical properties of silicon are listed in table 1.

\section{Results}

Calculations have been performed for two cases, and the velocity, temperature, concentration fields and interface shapes are obtained. Since similar flow patterns, isotherms and interface shapes have been presented previously $[17,20]$, the isotherms in the solid regions and melt zone are not given and only the flow patterns are kept for comparison.

Figs. 2 shows the distributions of flow patterns, concentration fields and the spatial concentration with segregation coefficient $K_{0}=0.5$ for different pulling rates. Corresponding results are listed from left to right for decreasing pulling rate relative to the heater. The global body of the liquid bridge shifts toward the single crystal rod, similar features have been noted in refs. [17], [18] and [20]. The results show that the free surface length has only a small change in $z$-direction; however, the shapes of the melt/multicrystal and the melt/single-crystal interfaces change significantly and the configuration of the liquid bridge is more non-symmetric if the pulling rate is larger. The vortex cell in the neighborhood of the melting boundary becomes smaller and weaker while the other cell near the solidification boundary becomes larger and stronger due to the larger pulling rate, which changes obviously the global body of the liquid bridge and the shapes of the interfaces. Furthermore, the concentration field is influenced by the convection in the melt zone and by the shapes of the interface and changes significantly for larger pulling rate in comparison with that for lower pulling rate. In topology, the iso-concentration lines seem to be symmetrical relative to the plane $z=0$ in the case of low pulling rate $[4,7]$ and more asymmetrical in case of larger pulling rate. It can be seen from fig. 2 that the concentration value is less, but not the least in the mixed core, which occurs at melt/feed interface and moves from the free surface to the symmetric axis for decreasing pulling rate. It is induced by the variation of the convection and interface shape for different pulling rates. The concentration field has uniform core of melting liquid and steep concentration gradients along the interfaces of phase changes in larger pulling rate, for example 


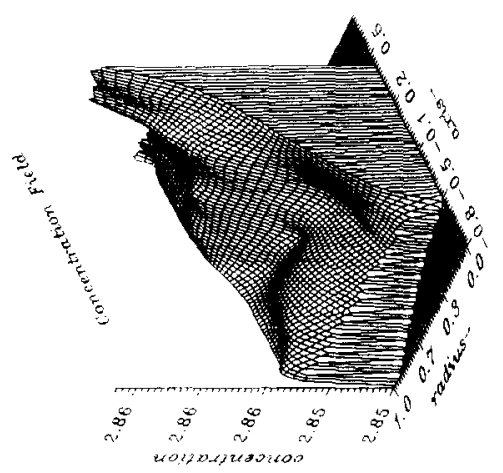

흐
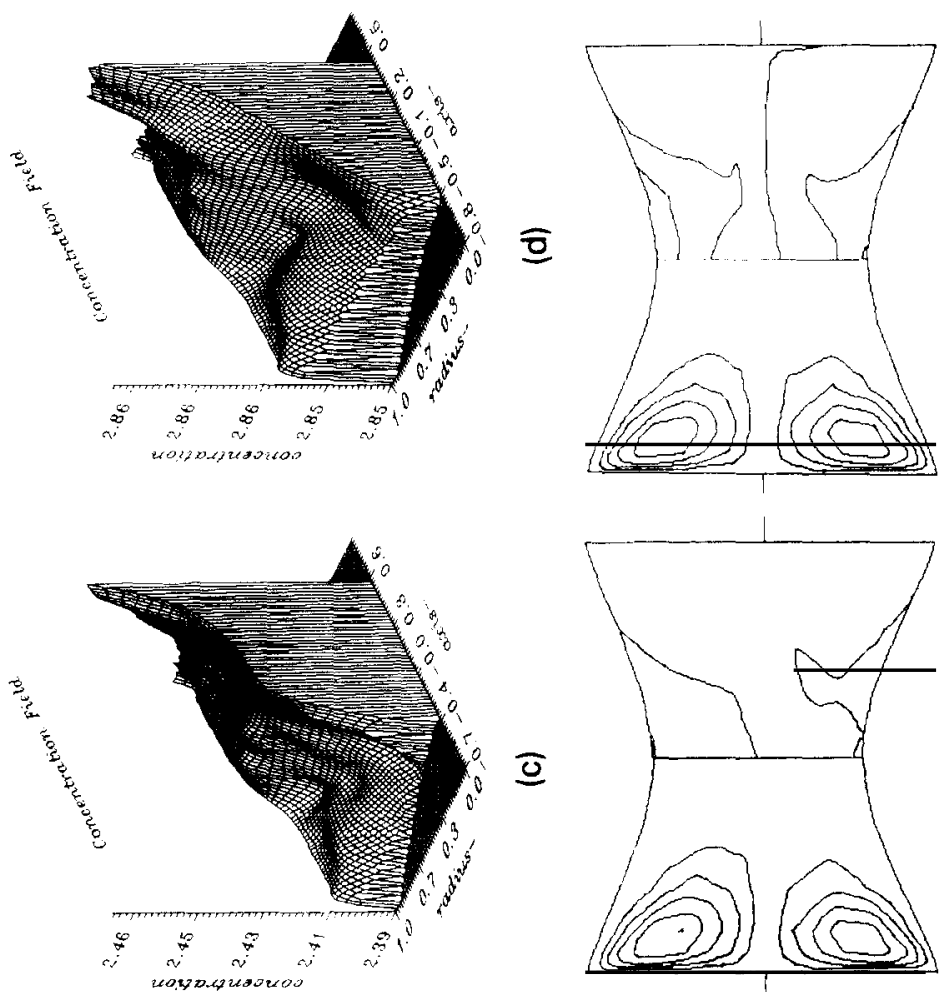

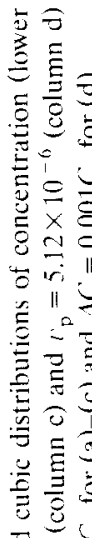

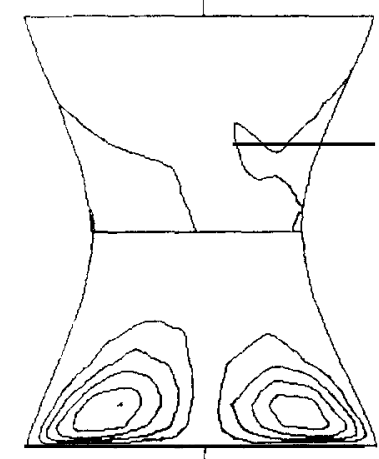

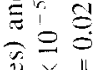

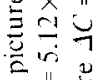

范。

$\div=$

影苛

可

0

学:
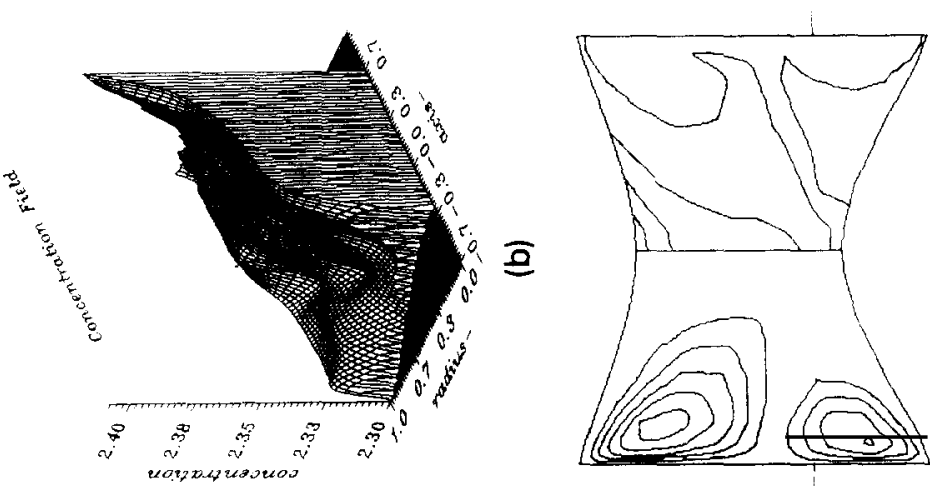

ธำ

$\stackrel{\Xi}{ }=0$

的

可

要

कि

至是

a $x$

पूत

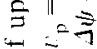

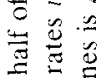
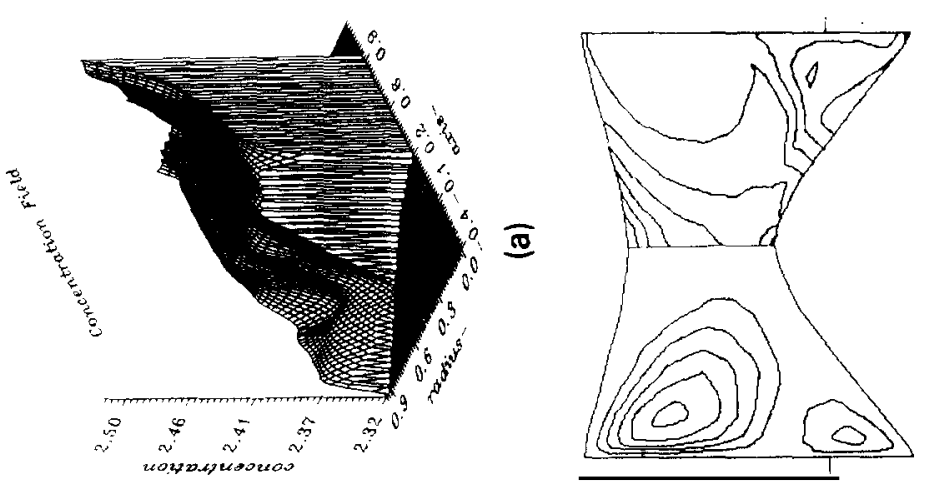

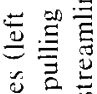

总

$\stackrel{5}{\underline{D}}$

莺

$\overline{\overline{0}}$

过

至

린

苟

i

这 


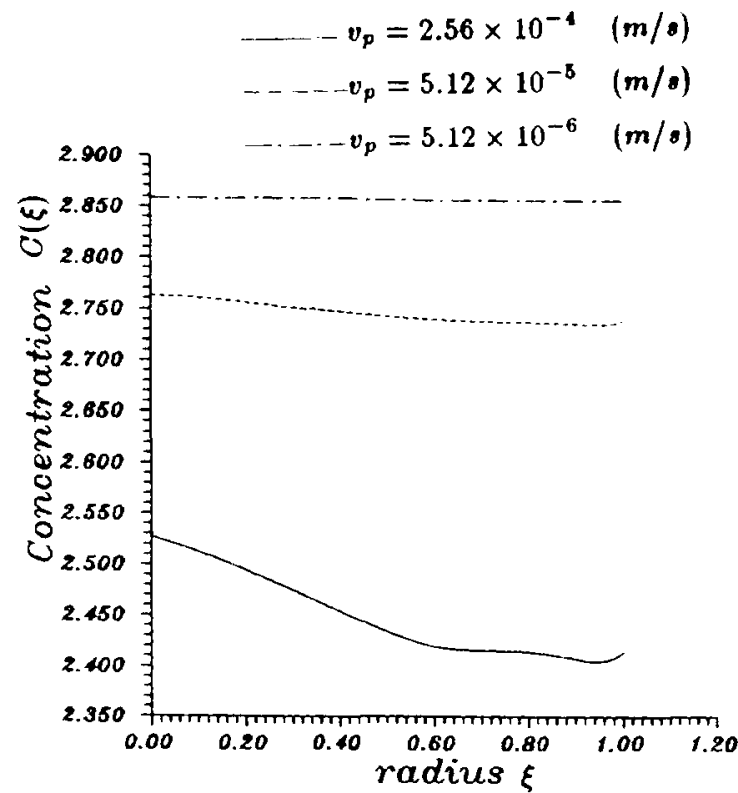

Fig. 3. Dopant concentration profiles in the radial direction along the melt $/$ crystal interface depending on different pulling rates for $K_{0}=0.5$.

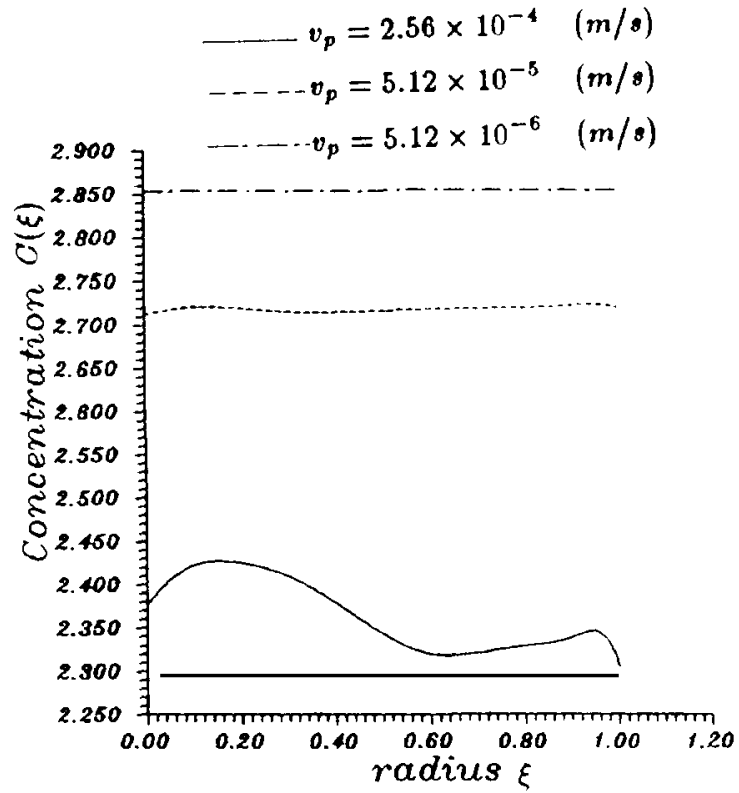

Fig. 4. Dopant concentration profiles in the radial direction along the melt/multi-crystal interface depending on different pulling rates for $K_{0}=0.5$.

$v_{\mathrm{p}}=2.56 \times 10^{-4} \mathrm{~m} / \mathrm{s}$. It is shown that the melt is mixed well, and this conclusion is similar to that noted in the BPS model $[2,5]$.

The radial distributions of concentration along the melt and solid interfaces and longitudinal distributions along free surface and along symmetric axis are given respectively in figs. $3-6$. In order to make the results clear, as shown in figs. 3 and 4, the concentration distribution is relatively uniform along the melt/solid interfaces in case of the lower pulling rate. For larger pulling rate, for example, $v_{\mathrm{p}}=2.56 \times 10^{-4} \mathrm{~m} / \mathrm{s}$, the concentration value along the melt/single-crystal interface decreases with the increasing radius $r$. The results show the lateral segregation which has larger radial variation for larger pulling rate, as shown in fig. 3 . This is different from the one for low pulling rate $[4,7]$. We can find the different tendencies of the concentration distributions along melt and solid interfaces in different pulling rates, as shown in fig. 2 on the spatial concentration distribution. So we can conclude that the dopant segregation degree for larger pulling rate is stronger than that for lower pulling rate. On the other hand, the concentration in multi-crystal is given by the sample; however, the boundary condition at melt/feed rod interface is given by the concentration conservation condition, a discontinuity appears at the boundary [4], and the concentration difference between melt and feed on the melt/feed interface is larger for lower pulling rate than for larger pulling rate, as shown in fig. 4. The longitudinal segregation along the free surface and along the symmetric axis in $z$-direction are shown in figs. 5 and 6 , respectively. The results illustrate that the longitudinal segregation depends strongly on pulling rate: the stronger the segregation, the larger the pulling rate.

However, the simulation of concentration distribution is more complicated. Fig. 7 shows the dependence of concentration maximum on pulling rates, and the profiles do not show monotonic features. The concentration maximum in the melt zone reaches the smallest value when the pulling rate is about $1.5 \times 10^{-4} \mathrm{~m} / \mathrm{s}$, and there are two peaks at $v_{\mathrm{p}}=0$ and $2.0 \times 10^{-4} \mathrm{~m} / \mathrm{s}$. The results should be analyzed further. 


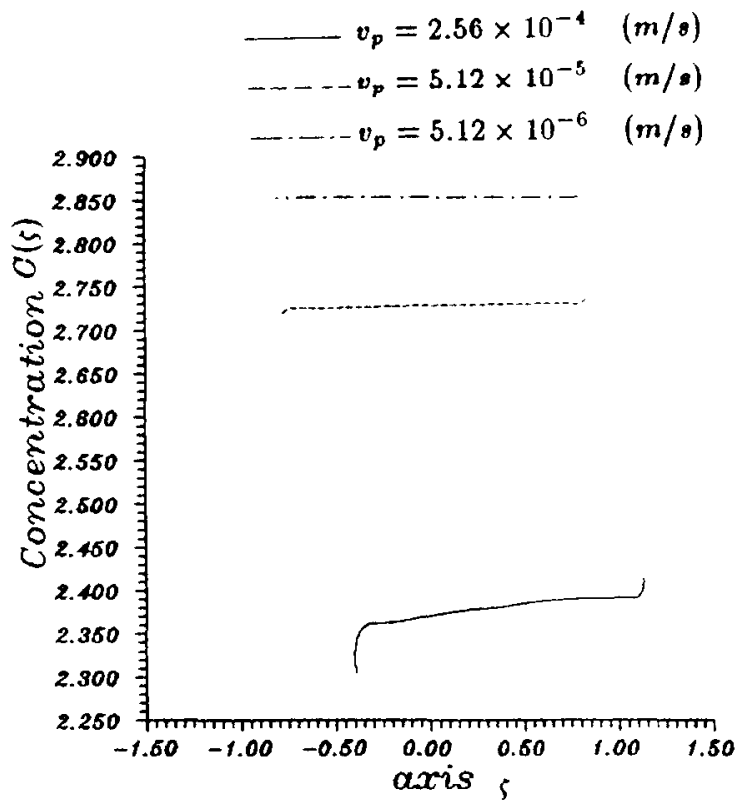

Fig. 5. Dopant concentration profiles in the axial direction along the free surface depending on different pulling rates for

$$
K_{0}=0.5 \text {. }
$$

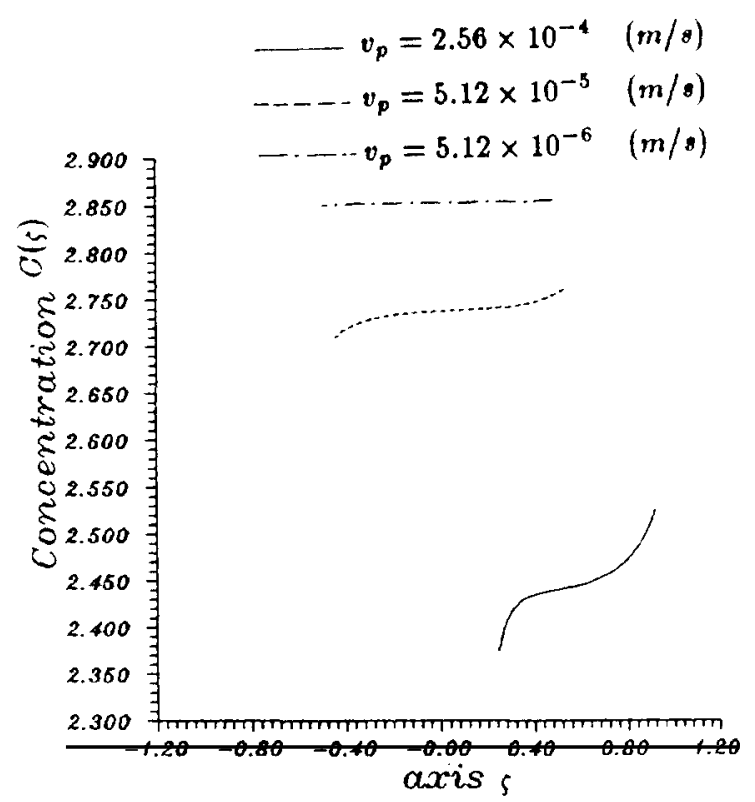

Fig. 6. Dopant concentration profiles in the axial direction along the symmetrical axis depending on different pulling rates for $K_{0}=0.5$.

We can discuss the effect of pulling rate in a simple case without convection; the problem can be treated with a one-dimensional model, and the following governing equations and boundary conditions are obtained:

$$
\begin{aligned}
& \operatorname{Pe}_{\mathrm{s}} V_{\mathrm{p}} \mathrm{d} C / \mathrm{d} \zeta=\mathrm{d}^{2} C / \mathrm{d} \zeta^{2} \\
& \zeta=0: \quad \mathrm{d} C / \mathrm{d} \zeta=\operatorname{Pe}_{\mathrm{s}} V_{\mathrm{p}}\left(C-C_{\mathrm{s}}\right), \\
& \zeta=L: \quad \mathrm{d} C / \mathrm{d} \zeta=\operatorname{Pe}_{\mathrm{s}} V_{\mathrm{p}}\left(1-k_{0}\right) C,
\end{aligned}
$$

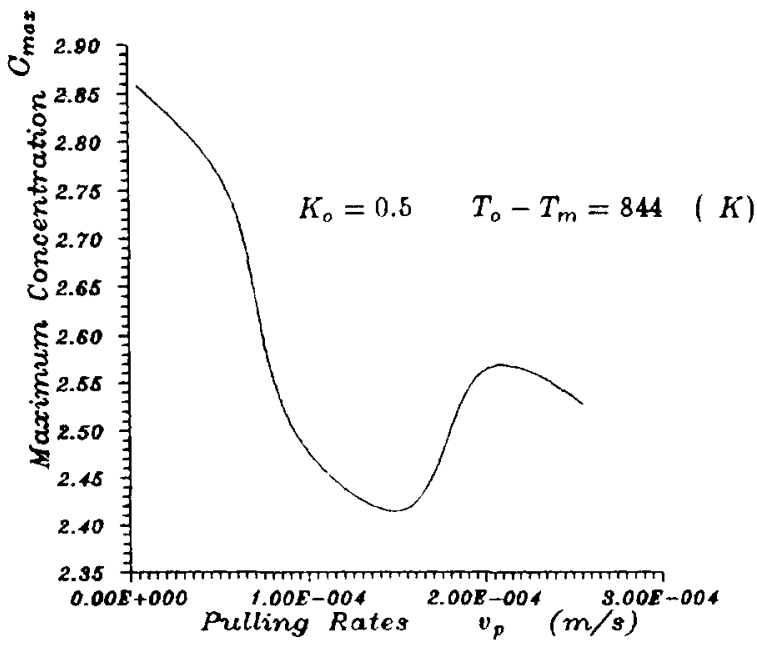

Fig. 7. Variation of concentration maximum in the liquid bridge depending on different pulling rates. 

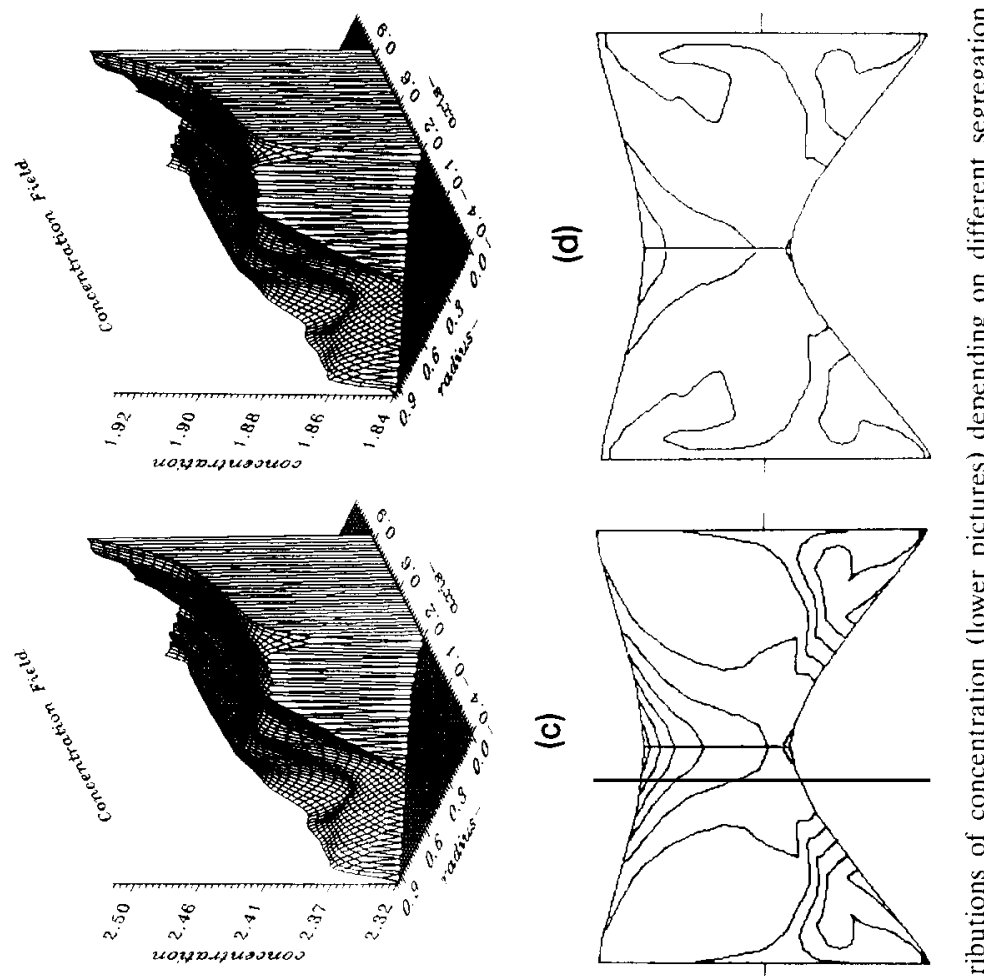

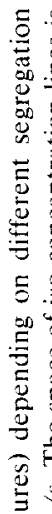
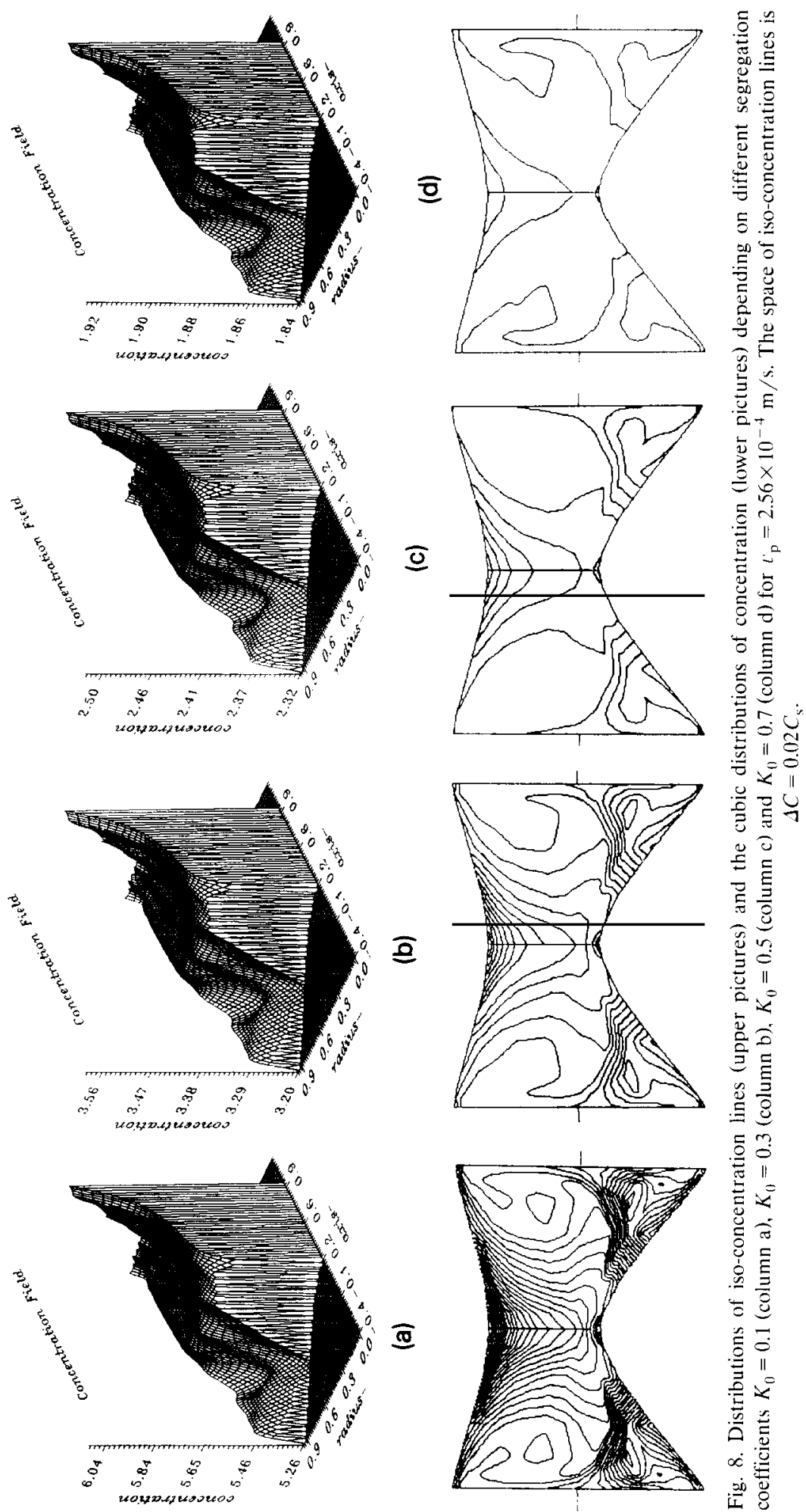

参讨

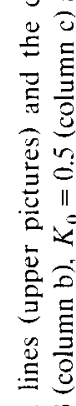

을

焉

过

竞

$\div$

咅 总

弯

$\infty$

数变 


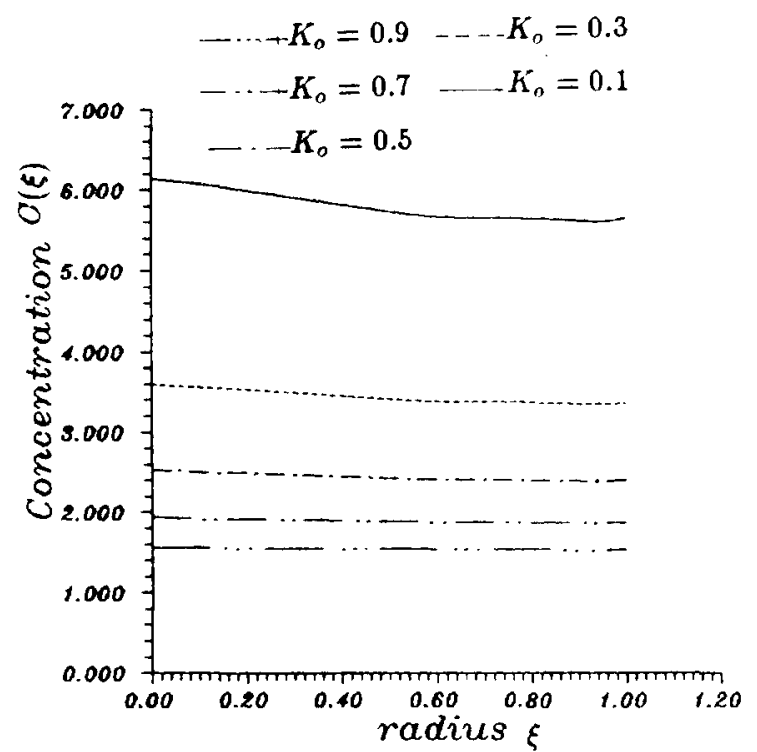

Fig. 9. Dopant concentration profiles in the radial direction along the melt/single-crystal interface depending on different segregation coefficients for $l_{\mathrm{p}}=2.56 \times 10^{-4} \mathrm{~m} / \mathrm{s}$.

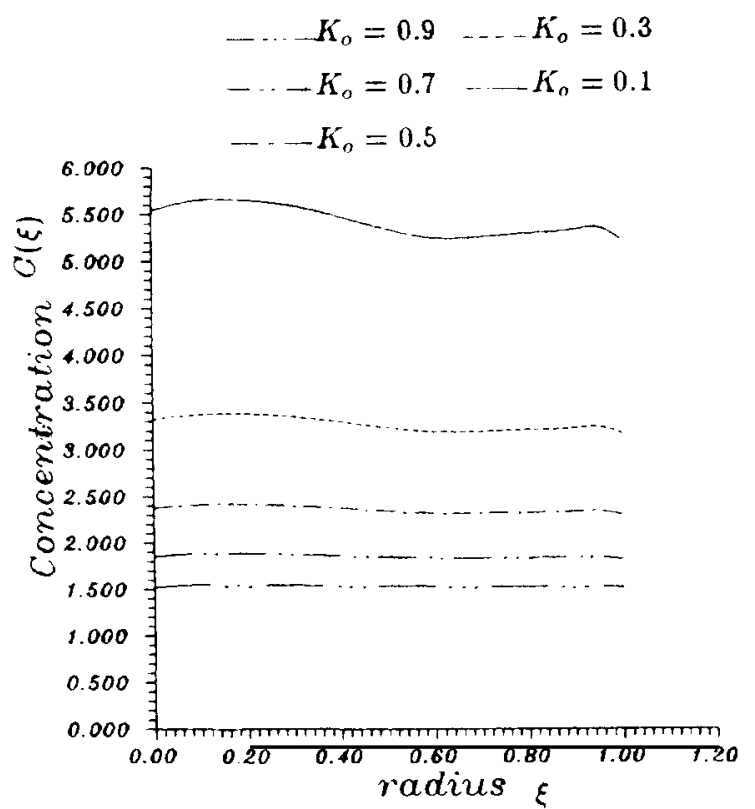

Fig. 10. Dopant concentration profiles in the radial direction along the melt/multi-crystal interface depending on different segregation coefficients for $v_{\mathrm{p}}=2.56 \times 10^{-4} \mathrm{~m} / \mathrm{s}$.

where $V_{\mathrm{p}}$ and $L$ denote the dimensionless pulling rate and the length of the melt zone, respectively; the other symbols are dimensionless and are defined as usual. The analytical solution is then given as

$$
C(\zeta)=\frac{\left(1-K_{0}\right) C_{\mathrm{s}} \exp \left(\mathrm{Pe}_{\mathrm{s}} V_{\mathrm{p}} \zeta\right)}{K_{0} \exp \left(\mathrm{Pe}_{\mathrm{s}} V_{\mathrm{p}} L\right)}+C_{\mathrm{s}}
$$

In comparison with the one-dimensional and two-dimensional models, let us adopt the same parameters, i.e., $\mathrm{Pe}_{\mathrm{s}}=10^{4}, V_{\mathrm{p}}=10^{-4}, K_{0}=0.5, C_{\mathrm{s}}=1.0$ and $L=1.0$. Then we obtain $C(\zeta) \in[1.368,2]$ for the one-dimensional model, which conclusion differs from $C>2$ in the two-dimensional model. This difference shows the influence of Marangoni convection on the concentration distribution. If $V_{\mathrm{p}}$ in the one-dimensional model varies from negative to positive, it could be imaged that the one-dimensional concentration $C(\zeta)$ may be greater than 2.0 .

Now, we will discuss the influence of different segregation coefficients $K_{0}$ on the fields in the melting zone, the steady state concentration fields and the spatial concentration distribution are shown for pulling rate $l_{\mathrm{p}}=2.56 \times 10^{-4} \mathrm{~m} / \mathrm{s}$ in fig. 8 . It can be concluded from the results that the concentration fields with the same pulling condition have similar topographies, although the segregation coefficients are different. The reason is that the convection has a similar effect on the concentration fields. The concentration mixed cores appear at the same position for different $K_{0}$ but they have distinct levels; for example, $C=1.515-1.555$ if $K_{0}=0.9$ and $C=5.236-6.143$ if $K_{0}=0.1$.

With a given pulling rate $v_{\mathrm{p}}=2.56 \times 10^{-4} \mathrm{~m} / \mathrm{s}$, the lateral segregation along the melt $/$ single-crystal and the melt/multi-crystal interfaces for different segregation coefficients $K_{0}$ are illustrated in figs. 9 and 10 . The segregation degrees are identical with variant tendency of the segregation coefficient $K_{0}$. There is a stronger segregation if $K_{0}$ is larger. The effect of Marangoni convection on segregation level is obvious, e.g. it mixes the melt well or weakens the concentration gradient in the melt zone. 


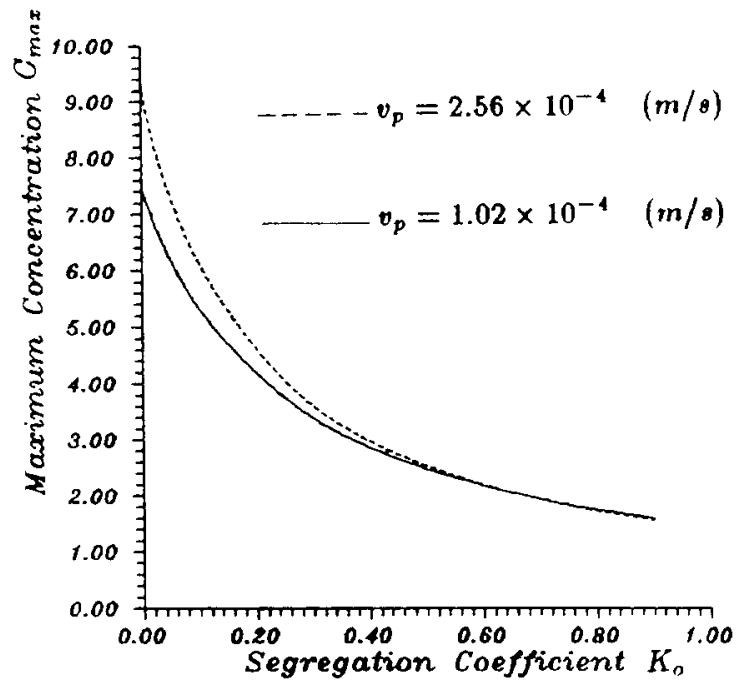

Fig. 11. Variation of maximum concentration in the liquid bridge depending on different segregation coefficients.

Additionally, it reduces the concentration level which ought to be higher without convection in the melt zone.

The maximum concentration profile with variation of $k_{0}$ is shown in fig. 11 . The results show that $C_{\max }$ is not inversely proportional to $K_{0}$, or, in other words, the effective segregation coefficient is less than the equilibrium segregation when $k_{0}$ is large and the effective segregation is larger than the equilibrium segregation when $k_{0}$ is small.

\section{Conclusions}

In the present paper, the effect of melt/solid phase change, the thermal and solutal diffusions, and the thermocapillary convection on the concentration distribution in melt zone is discussed for the steady process of crystal growth in the floating zone under zero-gravity condition. It has been shown that the convection driven by solutal capillary has little influence on melt convection for most cases of typical parameters, so its influence has not been considered in the present paper [4].

A finite element method is applied to iterate the phase change interfaces, concentration convection induced by thermocapillary surface tension and the melt flow. Comparing the existing results with the previous ones $[4,20]$ proves that our procedure is successful.

It could be concluded from the numerical results that the shapes of phase change interfaces, in addition to the thermocapillary driven convection, have significant influence on the concentration distribution in the melt zone. Convection makes the concentration distribution more uniform, but induces the lateral segregation at the same time. The effect of the pulling rod makes steep concentration gradients along the phase change interfaces and causes the "non-symmetry" of the concentration field. In general, the thermocapillary convection and the phase change convection have significant influence on concentration distribution. 


\section{Nomenclature}

$\mathrm{Bi}, \mathrm{Bi}_{1}, \mathrm{Bi}_{2}$

Bo

$c, C$

$C_{p}$

$C_{\text {s }}$

$D$

$f(\zeta)$

$g$

$\Delta H_{1}, \Delta H_{2}$

$K, K_{1}, K_{2}$

$K_{0}$

L

Ma

n

$N_{i}^{(e)}$

$p, P$

$\mathrm{Pe}_{1}, \mathrm{Pe}_{2}$

$\mathrm{Pe}_{\mathrm{s}}$

$r, r_{1}, r_{2}$

$r_{0}$

$\mathrm{Re}$

$z=S_{1}(r), z=S_{2}(r)$

$\mathrm{St}_{1}, \mathrm{St}_{2}$

$T, T_{1}, T_{2}$

$T_{\mathrm{m}}, T_{0}, T_{\infty}, T_{\mathrm{m}}^{*}, T_{0}^{*}$

$\Delta t$

$u, U$

$l_{\mathrm{p}}, V_{\mathrm{p}}$

$w, W$

$z$

$\alpha$

$\beta$

$\delta_{1}, \delta_{2}$

$\delta_{\mathrm{s}}$

$\epsilon_{1}, \epsilon_{2}$

$\epsilon^{*}$

$\zeta$

$\Theta, \Theta_{1}, \Theta_{2}$

$\mu$

$\nu$

$\xi$

$\rho, \rho_{1}, \rho_{2}$

$\sigma_{\tau}$

$\psi$

$\Omega$
Biot number in melt region, in single-crystal region and in multi-crystal region Bond number

Dopant concentration

Heat capacity

Dopant concentration in multi-crystal region

Dopant diffusivity

Distribution function of thermal radiation

Gravitational acceleration

Fusion heat of single-crystal and of multi-crystal

Thermal conductivity of melt, of single-crystal and of multi-crystal

Dopant segregation coefficient

Length of the whole region

Marangoni number

Unit vector normal to interface

Shape function

Pressures in melt region

Thermal Peclet numbers

Concentration Peclet number

Coordinates in radial direction

Radius of liquid bridge

Reynolds number

Interface position equations

Stefan numbers

Temperature in melt, in single-crystal and in multi-crystal region

Dimensionless temperatures

Pseudo-time interval

Flow velocities in $r$ direction

Pulling rates

Flow velocities in $z$ direction

Coordinate in axial direction

"Up-wind" corrector

"Up-wind" corrector

Dimensionless parameter $\mathrm{s}$

Dimensionless parameter

Relative density differences

Stefan-Boltzmann constant

Dimensionless coordinate in $z$ direction

Dimensionless temperatures

Viscosity

Kinetic viscosity

Dimensionless coordinate in $r$ direction

Density of melt, of single-crystal and of multi-crystal

Thermal coefficient of surface tension

Streamline function

Vorticity 


\section{References}

[1] W.G. Pfann, Trans. AIME 194 (1952) 747.

[2] G. Müller, in: Crystals: Growth, Properties and Applications, Vol. 12 (Springer, Berlin, 1988).

[3] Ch.J. Chang and R.A. Brown, J. Crystal Growth 63 (1983) 343.

[4] R.R. You and W.R. Hu, IAF-91-395, AIAA, 1991.

[5] J.A. Burton, R.C. Prim and W.P. Slichter, J. Chem. Phys. 21 (1953) 1987.

[6] J.J. Favier and L.O. Wilson, J. Crystal Growth 58 (1982) 103.

[7] Ch.E. Chang and W.R. Wilcox, Intern. J. Heat Mass Transfer 19 (1976) 355.

[8] G.M. Harriott and R.A. Brown, J. Fluid Mech. 126 (1983) 269.

[9] S.A. Nikitan, V.I. Polezhayev and A.I. Fedyushkin, J. Crystal Growth 52 (1981) 471.

[10] H.M. Ettouney and R.A. Brown, Comput. Phys. 49 (1983) 118.

[11] Ch.E. Chang and W.R. Wilcox, Intern. J. Heat Mass Transfer 9 (1976) 1976.

[12] W.R. Hu and Z.M. Tang, Scientia Sinica A 2 (1990) 169.

[13] Ch.H. Chun, J. Crystal Growth 48 (1980) 600.

[14] Z.H. Cao, J.C. Xie, Z.M. Tang and W.R. Hu, Scientia Sinica A 9 (1991) 964.

[15] B. Feuerbacher, H. Hamacher and R.J. Naumann, Materials Science in Space (Springer, Berlin, 1988).

[16] J. Zheng, Y. Kamotani and S. Ostrach, AIAA 90-0318, 28th Aerospace Science Meeting, Reno, NV, Jan. 1990.

[17] B. Xiong and Z.M. Tang, Microgravity Sci. Technol. 2 (1992), in press.

[18] C.W. Lan and Sindo Kou, J. Crystal Growth 108 (1991) 351.

[19] J.Y. Murthy and P. Lee, J. Heat Mass Transfer 31 (1988) 1923.

[20] B. Xiong and W.R. Hu, J. Semiconductors, submitted.

[21] S. Ostrach, Ann. Rev. Fluid Meth. 14 (1982) 313.

[22] Ke Cong Zhang, Fundamentals of Modern Crystals (Science Publ. Hall, Beijing, 1987).

[23] J.C. Heinrich, Intern. J. Numer. Methods Eng. 20 (1984) 447.

[24] J.C. Heinrich, P.S. Huyakorn and O.C. Zienkiewicz, Intern. J. Numer. Methods Eng. 11 (1977) 131. 